Meta

Journal des traducteurs

Translators' Journal

\title{
Vers une terminologie musicale afro-américaine : problèmes, techniques, solutions
}

\section{André Prévos}

Volume 32, numéro 3, septembre 1987

La fertilisation terminologique dans les langues romanes

URI : https://id.erudit.org/iderudit/003868ar

DOI : https://doi.org/10.7202/003868ar

Aller au sommaire du numéro

Éditeur(s)

Les Presses de l'Université de Montréal

ISSN

0026-0452 (imprimé)

1492-1421 (numérique)

Découvrir la revue

Citer cet article

Prévos, A. (1987). Vers une terminologie musicale afro-américaine : problèmes, techniques, solutions. Meta, 32(3), 306-313. https://doi.org/10.7202/003868ar d'utilisation que vous pouvez consulter en ligne. 


\title{
VERS UNE TERMINOLOGIE MUSICALE AFRO-AMÉRICAINE : PROBLÈMES, TECHNIQUES, SOLUTIONS
}

\author{
ANDRÉ PRÉVOS \\ The Pennsylvania State University, \\ The Worthington Scranton Campus, Dunmore, USA
}

Depuis la création de l'Académie, ou tout au moins depuis la parution de son premier dictionnaire en 1694 en particulier, l'évolution de la langue française a souvent été à l'origine de mouvements dont les initiateurs se voulaient militants soit en faveur de la prolongation du statu quo linguistique, soit en faveur d'une nécessaire adaptation trop longtemps évincée et menaçant l'aspect utilitaire de la langue'. Aujourd'hui, force nous est de constater que l'évolution de la langue est généralisée et que le langage évolue sous toutes ses formes : le changement dans les mots ou dans les sens de mots reflète toujours des réalités nouvelles ou des concepts jusqu'alors inusités ${ }^{2}$. Toutefois, ces changements, de par leur amplitude et leur résonnance culturelle, sont si rapidement ancrés dans le lexique que même les efforts officiels les plus exacerbés semblent ne produire que des résultats limités ${ }^{3}$.

Des commissions successives se sont principalement penchées sur le problème de la terminologie technique - souvent celle associée aux techniques dites " de pointe " et n'ont qu'exceptionnellement poussé leurs efforts vers les aires langagières plus axées sur l'art et la société. C'est à la fois à des arts et à des sociétés étrangères que le présent essai est consacré : aux formes musicales afro-américaines - jazz et blues en particulier - et aux problèmes terminologiques qui y sont associés, tant du point de vue technique (en ce qui concerne essentiellement le côté musical et musicologique), que du point de vue linguistique et stylistique (en ce qui concerne les paroles des chansons de blues en particulier). En effet, les paroles des blues ont toujours constitué le second maillon d'un assemblage quasi indissociable qu'elles forment avec la musique. De plus, les études linguistiques les plus avancées ${ }^{4}$, aux considérations significativement influencées par les soucis sociologiques ou culturels de leurs auteurs ${ }^{5}$, fournissent une incontestable preuve de la résilience de cette association.

Les Européens, et les Français en particulier, avaient pu prendre contact avec les productions artistiques afro-américaines dès les dernières décennies du dix-neuvième siècle, lorsque des groupes vocaux noirs américains spécialisés dans les gospels (un terme musical adopté sans effort de traduction) vinrent en Europe. Des groupes tels que les Fisk Jubilee Singers ou les Hampton Institute Singers furent réellement appréciés des audiences choisies qui eurent l'occasion de les écouter, mais on ne peut dire que le gospel devint très populaire ${ }^{6}$. Ce n'est qu'à partir du début de notre siècle que la musique afro-américaine va avoir un impact notable en France - avant la première guerre pour le ragtime et à partir de 1918 pour les autres styles. Le cas du ragtime illustre le degré de résistance linguistique et sociale propre à l'époque. Le mot fut traduit en français de la manière la plus littérale possible par «temps de chiffon ». À cette musique au nom sur- 
prenant fut associée une danse, la " danse du chiffon ", ainsi décrite par un artiste de music-hall (un autre mot directement emprunté - mais pas typiquement afro-américain) :

Un deux trois!

On lance le ventre en avant

En t'nant les bras ballants

Comme un orang-outang?.

La popularité du " temps du chiffon " allait certes diminuer, mais elle ne s'effaça totalement que lorsque apparurent des formes plus indigènes de la musique afro-américaine, immédiatement après la fin des hostilités, lorsque les orchestres noirs de l'armée américaine (en particulier celui du $158^{\mathrm{e}}$ régiment d'infanterie sous la direction de Jim Europe) permirent aux Parisiens d'entendre des airs afro-américains interprétés par des Noirs. L'entre-deux-guerres allait marquer l'avènement du jazz en France 8.

Tout d'abord, les termes " jazz » et " blues » furent adaptés directement et devinrent des mots français. Il était pratiquement impossible de dériver même la plus élémentaire traduction du premier, et une translittération du second n'aurait rien donné non plus. Toutefois, dans le cas du blues : le morceau "St Louis Blues » devint " La tristesse de St-Louis " par exemple?. Ensuite, d'autres tentatives furent lancées afin de trouver des traductions pour des expressions telles que " to have the blues ", "to feel blue ", "to be blue ». Si l'expression " avoir les bleus » ne signifie pas grand chose, "avoir le cafard" ou « broyer du noir " (si le calembour involontairement sinistre est oublié) sont plus acceptables $^{10}$. Quant aux formes musicales qui se firent jour au cours des décennies de l'après-guerre, elles aussi furent directement intégrées dans le vocabulaire français. C'est ainsi qu'après le "swing", on vit apparaitre le "be-bop", le " hard bop ", le " jazz rock ${ }^{11}$, pour le be-bop, l'emprunt est la seule solution car les syllabes be-bop ne voulaient rien dire au départ (un peu comme le terme artistique "dada " en France). Pour les autres, le terme " bop dur " serait encore instable ; le " jazz frais " semblerait pour le moins incongru (surtout qu'aujourd'hui le mot "cool " est bien inséré dans le vocabulaire populaire) ; quant au " jazz libre » il présupposerait un jazz qui ne le serait point! Le récent phénomène du " jazz fusion 》 nous ramène au problème antérieur : devonsnous ne traduire qu'une moitié du terme? (le problème peut paraître moins important $\mathrm{du}$ fait de l'exacte superposition orthographique des deux termes). Le cas du blues a conduit à de semblables emprunts. Tout d'abord on a parlé de blues " rural " ou " campagnard " pour le "rural blues" et le "country blues " (en français, la grande majorité des auteurs consultés considèrent le terme "blues " comme singulier et masculin), ensuite le "blues classique " des années vingt et trente, puis des blues " de la ville" et "urbains " pour les " city" et "urban blues »12. Là encore, les expressions sont plus proches de l'emprunt que de la traduction.

Lorsqu'on se tourne vers des termes plus techniques, les mêmes problèmes réapparaissent : doit-on simplement emprunter le mot original ou tenter de le traduire ? Prenons le cas du " growl » des musiciens du jazz. Les dictionnaires bilingues nous donnent des traductions centrées soit sur la notion de grondement et de grognement, soit celle de ronchonnement et de marmonnement. L'amateur de jazz sait bien que ces notions sont sous-jacentes lorsqu'un trombone ou un trompette (parfois même un saxophoniste ou un clarinettiste) joue avec cet effet de grincement affectant la sonorité de l'instrument qui, alors, grogne, ronchonne, gronde et marmonne mais qui aussi fait plus : il joue " growl ${ }^{13}$. Dans les exemples suivants tirés du vocabulaire du blues, les indications des dictionnaires sont encore moins utiles. Dire d'un guitariste de blues qu'il utilise la " technique du goulot de bouteille " peut demander de la part du lecteur non initié un effort similaire à la compréhension du terme "bottleneck technique » qui lui est préféré ; 
mais il ne sera point trop difficile d'imaginer qu'il s'agit de la technique qui consiste à utiliser un goulot de bouteille [bottle-neck] ou un objet métallique pour obtenir un effet de glissando sur la guitare ${ }^{14}$. Si on peut traduire le mot "flatpicking " par " jeu avec plectre " (qui est plus une explication qu'une traduction), il n'en demeure pas moins que traduire l'expression "finger picking " par " jeu en arpège" est encore moins satisfaisante ; car si le "finger picking " peut être un jeu en arpège, il peut aussi inclure une alternance ou une imbrication de basses et d'aiguës sans qu'il y ait d'arpèges ${ }^{15}$. Le cas des techniques d'harmonica nous fournit deux exemples problématiques : ce sont les termes techniques "choke " et "tongue tied", qui ne seront absolument point rendus par "bloqué " ou " étranglé " pour le premier et par " bloqué par la langue ", " obstrué par la langue ", ou "langue bloquante ". Ces deux mots indiquent des techniques permettant respectivement de bloquer avec la langue plusieurs trous de l'harmonica tout en soufflant ou en aspirant plus ou moins fort dans un autre trou de façon à transformer la note originale pour la faire monter d'un demi-ton ou d'un ton entier et de faire, ensuite, vibrer la langue avec un vibrato dans les trous bloqués, ce qui permet d'obtenir un " son de sonnette " en plus de la note jouée ${ }^{16}$.

Comme ces quelques exemples l'indiquent, les composantes techniques du vocabulaire de la musique afro-américaine exigent soit un emprunt direct soit une explication lexicale et ne se prêtent guère à la traduction. Comme nous le savons bien, dans la plupart des cas, la traduction technique ne prend en compte que des aspects n'ayant que peu (ou pas) de relations culturelles ou sociales dans les deux lexiques, celui de la langue de départ et celui de la langue d'arrivée (mais pouvant avoir des relations techniques proches). Dans le cas de la musique afro-américaine, nous devons tenir compte de la situation même de la société noire aux États-Unis (depuis les débuts de l'esclavage jusqu'à nos jours), tant du point de vue " technique " que du point de vue "social ". Les termes techniques du jazz et du blues ont principalement été créés hors des normes artistiques de la société blanche et ont donc un statut particulier même dans la langue angloaméricaine (c'est le cas des termes "jazz" et "blues » entre autres) ${ }^{17}$.

Dans la section consacrée aux paroles des blues, il va être possible de confirmer et d'illustrer ces particularités. Afin de constituer un échantillon de traductions suffisamment important et diversifié, on a décidé de consulter en priorité quatre ouvrages mettant en évidence les aspects divers et les problèmes variés que le traducteur doit prendre en compte. Ces ouvrages incluent un dictionnaire de la langue du blues, deux études françaises originales, et une traduction d'un ouvrage anglais ${ }^{18}$. De plus, afin de disposer d'un cadre organisationnel, il a été décidé de suivre la terminologie stylistique et lexicale de Vinay et Darbelnet ${ }^{19}$. Pour ces derniers, il y a sept procédés de traduction. Nous allons maintenant voir lesquels de ces procédés ont été utilisés de préférence par les auteurs et traducteurs considérés.

Le premier procédé est l'emprunt; le plus simple des procédés de traduction : il s'agit en effet de conserver le mot de la langue de départ, soit dans le but d'introduire une couleur locale, soit afin d'éviter les complications dues à la quasi-absence d'équivalents [V-D : 47]. Parmi les emprunts identifiés dans les paroles des blues traduits, il faut noter que deux de ces derniers ont été empruntés par l'anglais. C'est ainsi le cas de " chauffeur " [L:63] et de " hêsitation " [L: 153]. Les autres emprunts trouvés s'inscrivent aisément dans les deux catégories mentionnées ci-dessus. Pour la couleur locale nous pouvons mentionner " $\mathbf{H}$ " [L : 146] pour le haschish, "rocking chair " [S : 121] (l'expression " dodine " [B :243], traduction plus particulière sera trouvée plus tard), et "station" $[0: 74]$ au lieu de " gare ". En ce qui concerne la catégorie des quasi-absences d'équivalent, notons " jam session» $[L: 175]$, qui pourrait bien entendu être rendu par l'explication " réunion inopinée de musiciens jouant pour leur seul plaisir ", mais qui dé- 
truirait totalement l'idée originale ; un autre cas est «bo weevil» [O : 67] pour anthonome, un type de charançon du coton qui causa d'énormes ravages dans les plantations du sud des Etats-Unis entre 1900 et 1930. Il a été décidé de ne pas insister sur des emprunts tels que "dollar ", "sheriff ", et "whiskey ", qui sont rentrés dans le lexique français.

Le second procédé est le calque : on emprunte à la langue étrangère le syntagme, mais on traduit littéralement les éléments qui le composent. De même que pour les emprunts, il existe des calques anciens qui ont soit respecté les structures syntaxiques de la langue d'arrivée (c'est le cas de "compliments de la saison") soit introduit une construction nouvelle dans la langue (c'est le cas de "science fiction") [V-D : 47]. Les calques sont rares dans la langue et il n'a été possible d'en identifier qu'un ou deux. Le cas de « $J$ 'fais attention à mes 'P' et ' $Q$ ' » $[O: 21]$ pour l'anglais « $I$ ' $m$ minding $m y$ $P$ s and Qs ", parfois traduit par " je me surveille " ou "j'ouvre l'œil ", semble avoir été un choix délibéré du traducteur ; mais on ne peut affirmer sans ambiguité que le nouveau calque augmente la compréhension; le calque "boulet et chaîne " $[0: 167]$ pour "ball and chain " est plus heureux.

La traduction littérale au mot à mot désigne le passage de langue de départ à langue d'arrivée aboutissant à la fois à un texte correct et idiomatique sans que le traducteur ait eu à se soucier d'autre chose que des servitudes linguistiques [V-D : 48]. Ces deux procédés sont des solutions uniques, réversibles et complètes en elles-mêmes, la traduction littérale force souvent au recours à une traduction oblique. Dans les exemples suivants, deux niveaux ont été choisis, le niveau lexical et le niveau de l'arrangement. Au niveau lexical nous retrouvons " bo weevil" avec " anthonome " [L:37;O : 67] ; ainsi que "brother " rendu par " frère " [L : 48] (il est à noter que "sister " est souvent intraduisible et ne peut se rendre qu'exceptionnellement par "sœur"), "brownskin" rendu par " capresse" ou " capre " [B : 283] (aux Antilles, un capre est le rejeton d'un noir et d'une mulatresse). Au niveau de l'agencement (c'est-à-dire une traduction terme par terme sans changement de position), deux exemples ont été choisis, l'un d'eux ayant été choisi pour illustrer ce phénomème au niveau du dialecte (dans ce cas le cadjin de Louisiane) : "barrel of fun " rendu en cadjin par " barille d'agrément " [L : 55] (comme le célèbre " chien chaud " québécois), et "ants in my pants" rendu par "fourmis dans mes pantalons " AE : 287] ; dans ce cas il est évident que le texte français est à la fois correct et idiomatique.

La transposition est un procédé qui consiste à remplacer une partie du discours par une autre, sans changer le sens du message. Certaines transpositions sont obligatoires, d'autres sont facultatives [V-D : 50]. Les cas de "black cat bone" rendu par " os de chat noir " [L: 27] ou de "blue note " rendu par "note bleue " [L:33] illustrent la première catégorie, tandis que " deep sea diver" rendu par "plongeur de fond " [B : 286] l'amplifie. Mais nous savons aussi que le plongeur des paroles de blues ne plonge pas tout entier au fond de l'océan, et que l'expression est utilisée figurativement afin d'ajouter à l'imagerie textuelle de la chanson.

Le cinquième procédé est la modulation. C'est une variation dans le message, obtenue en changeant de point de vue ou d'éclairage. Une telle opération est justifiée quand on s'aperçoit que la traduction littérale ou même transposée aboutit à un énoncé grammaticalement correct mais qui se heurte au génie de la langue d'arrivée [V-D : 51]. Du point de vue lexical, le mot " cleanhead" rendu par " chauve " [L:58] ou " cocksuc$k e r$ " rendu par "lèche cul " [L:69], illustrent ce phénomène. Au niveau de l'agencement, "bloodhounds on my trail" rendu par "les molosses sont sur mes talons" [B : 265] ou par " les molosses sont à mes trousses " [O:80], ainsi que les deux autres exemples qui suivent : " red hot mama " rendu par « femme chauffée à blanc »[B : 287] (ce qui 
serait un comble pour des paroles chantées par un afro-américain !) et « to have his ashes hauled" rendu par "se faire ramoner la cheminée " [S : 133], montrent qu'au-delà de l'aspect linguistique, il est indispensable de prendre en compte d'autres facteurs qui ont marqué l'environnement du blues.

Il est parfois possible que deux textes rendent compte d'une même situation en mettant en ouvre des moyens stylistiques et des structures entièrement différents ; il s'agit alors d'une équivalence [V-D : 52]. Les équivalences sont le plus souvent de nature syntagmatique et intéressent la totalité du message ; il en résulte que la plupart font partie d'un répertoire phraséologique d'idiotismes, de clichés, de proverbes ou de locutions diverses. C'est en effet le cas pour les exemples trouvés : "Alabama" est rendu par "Alabama » [O : 77] (ce qui n'est pas une équivalence) mais aussi par «le sud » [L : 3] (qui en est une), " bone orchard " est rendu par " cimetière " $[\mathrm{L}: 37]$ et " cooling board" est rendu par " lit de mort " $[\mathrm{L}: 73]$. $\mathrm{A}$ ces équivalences lexicales, on peut ajouter, du point de vue de l'agencement, "give me long distance phone" rendu par "passe moi la province» $[$ B : 241].

Avec l'adaptation, le septième procédé, nous arrivons à la limite extrême de la traduction : il s'applique à des cas où la situation à laquelle le message se réfêre n'existe pas dans la langue d'arrivée et doit être créé par rapport à une autre situation qu'on juge équivalente $\{\mathrm{V}-\mathrm{D}: 52-53]$. Au niveau lexical on peut indiquer «back door man " rendu par " amant » [L: 11], "Charlie" rendu par " le blanc " [L: 63], "doghouse " rendu par "contrebasse " $[\mathrm{L}: 99]$ ou " yaller gal » rendu par « un café au lait » [B : 283]. Au niveau de l'agencement, nous pouvons mentionner : "big leg woman " rendu par " femme bien roulée » $[\mathrm{L}: 22]$; " to blow my horn " rendu par « dire ce que j'ai sur le coeur » $[\mathrm{L}: 31]$; " he sells as fast as the hog can chew his corn" rendu par " il le vend comme des petits pains " [S : 60] ; " to flag a ride " rendu par " arrêter une voiture " [S: 92] et "I'm down on you " rendu par " j'ai une dent contre toi » [S:144].

Parmi les sept procédés nommés ci-dessus, il est néanmoins nécessaire d'indiquer que, durant la recherche d'exemples, il a été plus facile de trouver des emprunts, des modulations et des adaptations, que des traductions littérales, des équivalences, ou des transpositions ; les calques ont été les moins faciles à trouver. Ces résultats ne prétendent point indiquer de tendances idéales mais, même avec un échantillon de petite taille, il est encore possible de voir que certains traits émergent : notamment l'importance respective des emprunts et des traductions littérales d'une part, et des adaptations et des équivalences d'autre part. Tout ceci semble s'accorder avec les remarques déjà formulées au sujet des termes techniques et de leur traduction.

Si la traduction des paroles de blues requiert l'utilisation des procédés ci-dessus, il n'en demeure pas moins que l'orientation stylistique souhaitée par le traducteur influence les choix de ce dernier. Ce phénomène est clairement illustré par les deux traductions d'un même blues; dans le cas présent, les quatre dernières strophes du "We Sure Got Hard Times » enregistré à Atlanta en 1930 par Barbecue Bob, et traduites par Springer et Gaille et Demêtre respectivement :

Tu t'es mis à chouraver, mais ça ne t'avance pas ( $x 2)$

Fais attention, tu vas te retrouver avec un boulet à la cheville.

Le saindoux et le bacon sont passés à un dollar la livre (x 2)

Le coton commence à se vendre mais il continue à baisser.

Juste avant les élections, tu disais comment tu allais voter ( $\mathrm{x} 2$ )

Et après les élections, on t'a vu la tête basse comme un chien battu. 
Les temps sont durs maintenant, c'est pas du bluff

Les temps sont durs, voilà ce qui nous arrive,

Pensez-y et repensez-y aujourd'hui, les temps sont durs ${ }^{20}$.

T'as commencé à chaparder mais ça ne sert pas à grand chose $(2 \mathrm{x})$

Prends biens garde à toi, tu te retrouveras le boulet et la chaîne au pied.

Le lard et le bacon sont à un dollar la livre (x 2)

On a commencé à vendre le coton mais il descend toujours plus bas plus bas.

Juste avant les élections tu racontais comment t'allais voter ( $\mathrm{x} 2$ )

Maintenant qu'elles sont passées, t'as la tête basse comme un vieux bouc.

Les temps sont durs, les temps sont durs, oui les temps sont vraiment durs maintenant Les temps sont durs, les temps sont durs, les temps sont vraiment durs maintenant

Eh bien t'as qu'à y penser et y penser encore, les temps qu'on vit sont durs maintenant ${ }^{21}$.

Il est facile de reconnaître certains emprunts (dollars, bacon), certains calques (boulet et chaîne), des traductions littérales (comme un vieux bouc), des modulations (chouraver/chaparder, ça ne t'avance pas/ça ne sert pas à grand chose), entre autres éléments communs aux deux traductions. Les différences n'en sont pas moins faciles à repérer : traduction littérale contre adaptation (vieux bouc : chien battu) par exemple, ou les différences de modulation dans la traduction de "talking» en dire et raconter. Mais ces différences d'ordre technique ne sont qu'une des composantes différentielles des deux textes. Les deux textes ont été arrangés de manière à refléter une certaine attitude socio-culturelle propre à l'interprète de blues dont les chansons étaient composées principalement pour un public noir.

Ces différences de traduction montrent combien il est malaisé de traduire des textes issus d'une couche sociale à statut particulier à l'intérieur d'une société déjà étrangère (le cas des Noirs dans la société américaine blanche), lorsque ses membres ont en commun une histoire, un art et une langue qui se sont formés hors des structures dominantes mais qui ont été néanmoins dominés ou influencés par ces dernières. Comme les travaux de Labov et de Kochman l'ont montré, la langue des Noirs américains est régie par des règles grammaticales et stylistiques particulières ${ }^{22}$. Ces facteurs doivent être et sont - pris en compte dans les deux traductions.

Les solutions à ce problème nous sont aussi suggérées par les deux exemples cidessus. Hors de la solution la plus triviale qu'est l'emprunt, ou de la plus extrême qu'est l'adaptation, le traducteur ayant affaire au vocabulaire musical afro-américain doit être bien informé des facteurs particuliers qui président (ou ont présidé) à l'élaboration de ce vocabulaire. Cet aspect est essentiel, car, hors de la traduction, c'est une équivalence entre deux textes qui est recherchée; et cette équivalence ne sera parfaite que lorsqu'elle reflétera une équivalence de situation. Le traducteur doit alors essayer de dégager la motivation profonde qui a poussé l'auteur du texte original pour la transposer dans la traduction ${ }^{23}$. Dans le cas présent, de nombreux éléments clairement étrangers à la culture américaine doivent être pris en compte. C'est de cet effort de compréhension de la société afro-américaine que peut provenir une meilleure traduction de ses productions musicales, tant du point de vue technique que du point de vue linguistique.

Notes

1. On pourra, par exemple, consulter l'ouvrage d'Etiemble, Parlez- vous franglais ?(1964 : Paris, Gallimard) et l'étude de Pierre Trescases, le Franglais vingt ans après (1982 : Montréal, Guérin), comme exemples du premier mouvement, et les nombreux magazines et revues spécialisées comme exemples du second mouvement. Dans le cas ces nouveaux mots créés par la vague informatique, on pourra voir en particulier : Eugene F. Gray (1985) : "French Computer Terminology ", French Review 58, pp. 805-810 et les sources qu'il mentionne. 
2. Voir, par exemple : Sophie Lannes et Hortense Chabrier (1986) : "Le français miroir des Français. L'Express va plus loin avec Alain Fantapié ", l'Express international, 4 avril, pp. 58-64.

3. Les succès pour le moins mitigés des Commissions de terminologie en sont l'illustration la plus nette

4. À ce sujet, on peut consulter, entre autres : Dennis Jarrett (1978) : "The Singer and the Bluesman : Formulations of Personality in the Lyrics of the Blues ", Southern Folklore Quarterly 42:1, pp. 31-38 ; Michael Taft (1978) : "Willie McTell's Rules of Rhyme : A Brief Excursion into Blues Phonetics", Southern Folklore Quarterly 42:1, pp. 53-72; Michael Taft (1983) : Blues Lyrics Poetry: An Anthology, New York, Garland Publishing Company ; Michael Taft (1984) : Blues Lyric Poetry : A Concordance, 3 vol., New York, Garland Publishing Company ; Jeff Todd Titon, Editor (1981) : Downhome Blues Lyrics. An Anthology from the Post World War II Era, Boston, Twayne Publishers ; Eric Sackheim, compiler (1969) : The Blues Line. A Collection of Blues Lyrics, New York, Grossman Publishers.

5. À ce sujet, on peut consulter, entre autres : Paul Oliver (1963) : The Meaning of the Blues, New York, Collier Books ; Barry Lee Pearson (1984) : "Sounds Good to Me ". The Bluesman's Story, Philadelphia, University of Pennsylvania Press; Jeff Todd Titon (1977) : Early Downhome Blues. A Musical and Cultural Analysis, Urbana, University of Illinois Press; David Evans (1982) : Big Road Blues. Tradition and Creativity in the Folk Blues, Berkeley, University of California Press ; Lawrence W. Levine (1977) : Black Culture and Black Consciousness, New York, Oxford University Press; Houston A. Baker, Jr. (1984) : Blues Ideology, and Afro-American Literature, Chicago, The University of Chicago Press.

6. Voir, par exemple : Levine, p. 116. La musique gospel sera redécouverte en France dans les décennies qui suivront la fin de la Première Guerre mondiale.

7. Dans : Ian Whitcomb (1974) : After the Ball. Pop Music from Rag to Rock, Baltimore, MD, Penguin Press Inc., p. 20.

8. Paul Yonnet (1985) : Jeux, modes et masses. La société française et le moderne. 1945-1985, Paris, Gallimard, pp. 144-155.

9. R[obert] P[ac] (1986) : "Amérique-France ", Différences 57/58, p. 25.

10. Voir à ce sujet l'éloquent commentaire de Jacques Réda dans son ouvrage : l'Improviste. Une lecture du jazz (Paris, Gallimard, 1980), pp. 14-15.

11. Pour les définitions de ces termes on pourra consulter le Dictionnaire du jazz de Hugues Panassié et Madeleine Gautier (Paris, Albin Michel, 1971).

12. Robert Springer (1985) : le Blues authentique. Son histoire et ses thèmes, Paris, Éditions Filipacchi, pp. $63-64,147-148,161$.

13. Pour une défnition plus élaborée, voir le Dictionnaire du jazz.

14. Pour une caractérisation plus détaillée, on peut consulter : Gérard Herzhaft (1984) : Nouvelle encyclopédie du blues, Paris, Jacques Grancher, pp. 21-22. De là est aussi dérivée l'expression "knife style » où un couteau de poche ou un canif joue le rôle du goulot de bouteille.

15. Voir, en particulier, Springer, p. 218

16. On pourra se reporter aux explications de l'harmoniciste de blues Billy Boy Arnold et aux commentaires de Benoit Blue Boy dans l'interview d'Arnold parue dans la revue française Soul Bag 107, pp. 6-8 (1986).

17. À ce sujet, on peut consulter : Albert Murray (1976) : Stomping the Blues, New York, McGraw Hill Book Company, pp. 57-76.

18. Les ouvrages mentionnés sont les suivants : Jean-Paul Levet (1986) : Talkin'That Talk. Le langage du blues et du jazz, Levallois-Perret, CLARB/Soul, identifié par [L:] ; Guy Claude Balmir (1982) : Du chant au poème. Essai de littérature sur le chant et la poésie populaires des Noirs américains, Paris, Payot, identifié par [B : ]; Robert Springer (1985) : le Blues authentique. Son histoire et ses thèmes, Paris, Éditions Filipacchi, identifié par [S : ]; Gilles Oakley (1985) : Devil's Music. Une histoire du blues, traduction de Hubert Galle. Traduction des lyrics revue par Jacques Demêtre, Paris, Édition Denoël, identifié par [O: ]. Dans le texte, l'initiale est suivie des numéros de page.

19. J.-P. Vinay et J. Darbelnet (1958) : Stylistique comparée du français et de l'anglais, Paris, Marcel Didier. Ouvrage identifié par [V-D : ] ; les initiales seront suivies par les numéros de page où ont été trouvées les indications.

20. Springer : pp. 120-121.

21. Oakley : p. 167.

22. Williams Labov (1972) : Language in the Inner City. Studies in the Black English Vernacular, Philadelphia, University of Pennsylvania Press; Thomas Kochman, Editor (1972) : Rappin' and Stylin' Out. Communication in Urban Black America, Urbana, University of Illinois Press.

23. Vinay et Darbelnet : p. 22 


\section{APPENDICE}

"We Sure Got Hard Times" : original et traductions

Springer : pp. 120-121

Tu t'es mis à chouraver, mais ça ne t'avance pas, (x 2)

Fais attention, tu vas te retrouver avec un boulet à la cheville.

Le saindoux et le bacon sont passés à un dollar la livre, (x 2)

Le coton commence à se vendre mais il continue à baisser.

Juste avant les élections, tu disais comment tu allais voter, (x 2)

Et après les élections, on t'a vu la tête basse comme un chien battu.

Les temps sont durs maintenant, c'est pas du bluff,

Les temps sont durs, voilà ce qui nous arrive,

Pensez-y et repensez-y, aujourd'hui, les temps sont durs.

Gakley : p. 167

T'as commencé à chaparder, mais ça ne sert pas à grand chose, (x 2)

Prends bien garde à toi, tu te retrouveras le boulet et la chaîne au pied.

Le lard et le bacon sont à un dollar la livre, (x 2)

On a commencé à vendre le coton, mais il descend toujours plus bas, plus bas.

Juste avant les élections, tu racontais comment t'allais voter, ( $\times 2$ 2)

Maintenant qu'elles sont passées, t'as la tête basse comme un vieux bouc.

Les temps sont durs, les temps sont durs, oui les temps sont vraiment durs maintenant

Les temps sont durs, les temps sont durs, les temps sont vraiment durs maintenant,

Eh bien t'as qu'à y penser et y penser encore, les temps qu'on vit sont durs maintenant.

"We Sure Got Hard Times" (Barbecue Bob, Atlanta, 1930)

You started in moochin', but your moochin's in vain, You started into moochin', but your moochin's in vain. Be careful with yourself, you'll get a ball and chain.

Lard and bacon gone to a dollar pound,

That lard and bacon gone to a dollar pound,

Cotton has started to sellin', but it keeps goin' down and down.

Just before election, you was talkin' how you was goin' to vote (x 2) After 'lection was over, your head's down like a billy-goat.

Hard time, hard time, sure got hard time now,

Hard time, hard time, we got hard time now,

Well, just think and think about it, we sure got hard time now. 